clinical skills e.g. airway adjuncts and managing emergencies as a team in the hospice. The session also highlighted other aspects of emergency care that would benefit from review, such as practical access to guidelines and emergency drug use. Conclusions High fidelity point of care simulation is a useful tool in palliative care MDT teaching in the hospice. It benefits those attending on a personal level and provides insight and safe 'real-time' practice for possible emergencies and can lead to positive changes to these systems.

\section{OPIOID CALCULATIONS VIA BISCUITS}

Elizabeth O'Brien, Lindy Brooks, Alicia Waite. Manchester Macmillan Support and Palliative Care Service - North Locality

\subsection{6/spcare-2020-PCC.53}

Background Specialist Palliative Care practitioners tend to have a familiarity with the mental gymnastics to calculate equipotent doses of analgesia and ensuring safe calculations is critical to medicines safety. All healthcare colleagues who manage medications should be aware of the relative strengths of strong opioids but achieving this with a wide range of learning styles can be a challenge. A hands on education module with a more visual component was developed to address aspects of the VARK learning styles for both district nurses and specialist colleagues.

Methods To develop practical and memorable education in opioid relative strengths a biscuit equivalence unit of $5 \mathrm{mg}$ oral morphine' was used. Commonly used opioids were identified and given to attendees in pairs to calculate how many 'biscuit equivalent units' the medication total daily dose represented. Actual wrapped biscuits were utilised (and eaten during the session) to show the relative strengths of common opioids ie morphine orally and via CSCI, fentanyl and buprenorphine patches and oxycodone.

Results The educational session has to-date been run 2 out of a planned 3 sessions with requests to provide at additional locations. All attendees have provided positive feedback and reflected that it was a novel way of understanding relative strengths of opioids. It provided the opportunity to rediscuss opioid strengths and the visual pile of biscuits, in particular for fentanyl patches, provided a very memorable point of reference.

Conclusion Understanding opioid doses and relative strengths of medications is a central aspect of safe medication management. Ways of making this more memorable for practitioners will improve their familiarity and safety in using these medications and supporting patients and families in their safe use. We often say that a cup of tea and a biscuit go a long way in conversation, their use in education may be just as effective.

\section{THE KNOWLEDGE, CONCERNS AND ATTITUDES OF PALLIATIVE HEALTH CARE PRACTITIONERS IN TREATING PATIENTS WITH SUBSTANCE USE DISORDER}

Natasha Palipane. Farleigh Hospice

10.1136/spcare-2020-PCC.54

Background In the UK, we are seeing an increase in the number of older drug users including those accessing drug treatment services. Because harm reduction interventions have resulted in more older drug users dying of non-drug related causes, studies suggest that they are likely to have a higher morbidity than the general population. Serving the palliative needs of those with substance use disorder (SUD) is often difficult due to the complexities of symptoms, their social circumstances and health care practitioner (HCP) understanding. A rapid evidence assessment in 2019 revealed limited inquiry into the experiences and attitudes of service providers.

Aim To evaluate the knowledge, concerns and attitudes of palliative HCP's (doctors, nurses and healthcare assistants) working in a range of clinical environments in treating patients with SUD in the Mid-Essex region.

Methods A mixed methods approach was carried out using an online survey tool. Quantitative evaluation of knowledge was measured using a 10-item true/false questionnaire. Confidence in managing patients with SUD was investigated using a Likert scale. Concerns relating to management were collated with a qualitative approach and interpreted by emergent coding analysis.

Results 40 health care practitioners (HCPs) working in the community, hospice inpatient unit or hospital completed the survey. $82 \%$ had experience in managing patients with SUD. Knowledge about medicine management and pain control was varied across the three roles. Although the majority felt confident in assessing pain in SUD, over 70\% lacked confidence in managing opioid substitution therapies. Confidence in planning discharge was also low. Frequently expressed concerns included tolerance to analgesia, unpredictable behaviour and safe drug storage.

Conclusions Despite the increasing palliative needs in SUD, there appear to be gaps in knowledge and low confidence towards management amongst HCP's. More support through education and policy is required at a local level.

\section{THE EDUCATION AND PRACTICE OF SELF-CARE IN PALLIATIVE MEDICINE TRAINEES, A QUALITATIVE STUDY}

Abi Ponnampalam, Adrienne Sharples. Farleigh Hospice, Chelmsford, University of Bedfordshire

\subsection{6/spcare-2020-PCC.55}

Context The emotional burden of regularly confronting suffering when caring for dying patients can affect the wellbeing of palliative care clinicians. Experienced clinicians recommend self-care strategies as a way of sustaining this work. There is increasing evidence for the effective use of self-care strategies in other caring professions who face similar challenges. Little is known about how doctors-in-training learn such skills. The aim of this study was to explore how trainees in palliative medicine learn and practice self-care strategies.

Methods Eight palliative trainees in one region in England participated in a qualitative study using semi-structured interviews. An inductive thematic approach was used to analyse the data.

Results Five closely linked themes are described detailing the perspectives of the trainees. Self-care was recognised as being integral to their identity as a palliative medicine clinician, even though it was not openly discussed. Trainees were keen 